\title{
Ontologías para modelar la Investigación Científica en Ingeniería Civil
}

\author{
Ontologies to model in Civil Engineering
}

\author{
M.Sc: Ingrid Durley Torres. PhD: Jaime Alberto Guzmán Luna. M.Sc: Mauricio López Bonilla \\ Facultad de Ingenierías, Institución Universitaria Salazar y Herrera, Medellín, Colombia \\ Facultad de Ingenierías, Universidad Nacional de Colombia, Medellín, Colombia \\ Departamento de Ciencias Básicas, Fundación Universitaria Luis Amigó, Medellín, Colombia \\ i.torreseiush.edu.co \\ jaguzman@unal.edu.co \\ mauricio.lopezbodamigo.edu.co
}

\begin{abstract}
Resumen- Con el ánimo de reutilizar las investigaciones previamente logradas, pero con el propósito de alivianar las tareas de los seres humanos-investigadores, se hace necesario normalizar en un modelo compartido las prácticas de laboratorio de manera que sea comprensible y reutilizable, por todos los investigadores que comparten el interés científico, en el ámbito de la ingeniería civil. Este trabajo se concentra en exponer el proceso de formalización del conocimiento científico, haciendo uso de ontologías, con el objetivo de hacer reutilizable la actividad experimental del campo de ingeniería civil.
\end{abstract}

Palabras clave - ontología, investigación científica, reutilización, experimentos, ingeniería civil

\begin{abstract}
- with the objective of reuse research previously achieved, but in order to lighten the tasks of human researcher's beings, all researchers it is necessary to standardize on a shared laboratory practices in a way that is understandable and reusable model they share a scientific interest in the field of civil engineering. This work focuses on exposing the formalization of scientific knowledge, using ontologies, with the aim of making the experimental reusable camp activity civil engineering.
\end{abstract}

Key Word - Ontology, scientific research, reuse, experiments, civil engineering.

\section{INTRODUCCIÓN}

Un protocolo representa una secuencia de operaciones que habitualmente son escritas en lenguaje natural [13]. Estos protocolos se presentan a manera de "receta" proporcionando paso a paso la descripción de procesos. Dicha secuencia de tareas y operaciones en la investigación experimental son consideradas unidades fundamentales de conocimiento [4].
"Los investigadores siguen y generan protocolos en sus actividades diarias, es así, como los protocolos experimentales reflejan las habilidades o conocimientos prácticos generados en un laboratorio, los cuales luego son compartidos y adaptados para diversos propósitos" [13]. Lo más importante, de los protocolos experimentales radica en su esencialidad para el avance de la investigación en un dominio contextual, toda vez, que se representan como piezas centrales en los esfuerzos de reutilización y reproducibilidad [8].

En este nuevo escenario cobra relevancia la necesidad de formalizar algunos aspectos claves de la ciencia en los distintos dominios reales de aplicación. En la actualidad los principales trabajos de formalización, se han concentrado en dominios como bioinformática [10], la astronomía [23], la biología [2, 17] y la genética [13], pero pese a los grandes esfuerzos los trabajos en otros campos son incipientes o nulos, tal es caso de la Ingeniería Civil; en cuyo desarrollo científico, los investigadores implementan protocolos, para documentar actividades experimentales en áreas como: concreto y pavimento, estructuras, hidráulica, suelos y geología física (por citar algunos). En cada una de esas áreas, un investigador define y realiza protocolos experimentales específicos que proporcionan información relacionada con los recursos usados, los datos empleados, las actividades ejecutadas, los éxitos o fracasos alcanzados, los tiempos consumidos, las fechas de registro, los resultados obtenidos y las conclusiones formuladas, junto con algunas otras anotaciones relacionadas. Sin embargo, tales experimentos son construidos bajo las consideraciones (mentales y de vocabulario) personales de cada individuo investigador, careciendo en la mayoría de los casos de una representación única formalmente estructurada, que permita compartir y reutilizar la valiosa información del protocolo experimental allí contenido. 
Con el objetivo de hacer explícito el conocimiento registrado en los protocolos experimentales del dominio de la ingeniería civil y lograr un mayor entendimiento por un grupo de individuos y por una máquina [18], este articulo describe el proceso de formalización semántica de un protocolo experimental del área de ingeniería civil, para que la interpretación y reutilización de la información experimental pueda ser soportado entre distintos individuos, incluyendo máquinas.

Para dar un mayor detalle del proceso este artículo se organiza de la siguiente manera: la sección II, presenta el marco conceptual relacionado con la temática; la sección III, recopila un análisis comparativo de las principales ontologías, usadas para describir los dominios experimentales; la sección IV, presenta un análisis del caso específico de ingeniería civil, y la propuesta de representación semántica; la sección $\mathrm{V}$, presenta las conclusiones y proyección futura, resultado de este trabajo y finalmente se presentan las referencias de los trabajos que de una u otra forma hicieron parte inspiradora de este artículo.

\section{MARCO CONCEPTUAL}

\section{A. Protocolos experimentales.}

"Un protocolo es una especificación planeada que tiene suficiente nivel de detalle y la información cuantitativa para comunicarla entre los expertos de dominio, de modo que diferentes expertos de dominio de forma fiable serán capaces de reproducir el proceso de forma independiente" [13].

Actualmente los protocolos experimentales de muchas áreas (entre ellas la civil), están registrados en su gran mayoría en unos documentos físicos, representados por una guía y un cuaderno de laboratorio. La guía, se refieren a un documento escrito que contiene instrucciones sobre cómo hacer ciertos procedimientos de laboratorio, con el propósito de conseguir resultados reproducibles independiente de quien los haga [18]. Los segundos, los cuadernos, constituyen un registro completo de todo el trabajo práctico experimental realizado [13]; tales cuadernos, contienen toda la información necesaria (operaciones, hechos observados y conclusiones), para que cualquier investigador pueda reproducir el trabajo realizado. Al ser llevado de forma manual, se espera que los cuadernos de laboratorio sean escritos de manera legible, ordenada y compresible. Estas condiciones operativas son primordiales, por ejemplo, en casos relacionados con interpretación y reutilización del experimento. Sin embargo, los protocolos experimentales, reportados en elementos físicos (guías y cuadernos) tienen limitaciones importantes [13] como la alta dificultad para compartir la información almacenada ya que los documentos físicos al ser construidos por distintas personas, varían en su organización, terminología y presentación, entre ellos, sin seguir un único estándar compartido. Una alternativa para mejorar este aspecto, es la construcción de una tabla de contenido, sin embargo, este procedimiento es ignorado por la mayoría de investigadores por el tiempo que debe invertirse en ello.

Bajo las condiciones actuales, interpretar o reutilizar las actividades experimentales del dominio de la ingeniería civil, obliga a quien quisiera usarlas a invertir altas cantidades de tiempo para descifrar el lenguaje usado por cada autor en sus documentos (aunque pertenezcan a la misma temática) y asociarlo a su interés investigativo, para poder especificar lo que resulta útil o no. Si a esto le sumamos la cantidad de documentos producidos por todos los autores, la tarea resulta tediosa, altamente costosa en recurso humano y muy lenta. Esta serie de desventajas exigen formular un modelo de representación formal, que normalice la práctica de los protocolos experimentales de ingeniería civil, haciéndolo comprensible para su reutilización.

\section{B. Ontología}

Una ontología es una especificación explicita de una conceptualización que permite una estructura y contenido explícito de una información de la realidad [9]; una conceptualización es una vista sencilla y precisa del mundo la cual se utiliza para representar el mundo para propósitos específicos. Explicito significa que los conceptos a utilizar sean detallados ya que esto ayudara a tener un mayor entendimiento de la realidad [15]. Esto también permite que la ontología pueda ser legible por una máquina y no solo sea aceptada por una persona sino también por un grupo de individuos. La ontología codifica reglas implícitas de la existencia, las reglas son independientes del dominio de la aplicación en las que se usen. Las otologías tratan de formar un esquema exhaustivo y riguroso en un domino dado, esto facilita la comunicación y compartición de información entre diversos sistemas [15].

Una ontología para describir un experimento científico es una especificación de forma concreta de una conceptualización, la cual proporciona la estructura y el contenido de forma suficientemente detallado, para describir un experimento [12].

\section{Ingeniería Civil}

La Ingeniería Civil es la disciplina de la ingeniería profesional que emplea conocimientos de cálculo, mecánica, hidráulica y física para encargarse del diseño, construcción y mantenimiento de las infraestructuras emplazadas en el entorno, incluyendo carreteras, ferrocarriles, puentes, canales, presas, puertos, aeropuertos, diques y otras construcciones relacionadas [22].

Existen varias ramas y subdisciplianas dentro de la ingeniería Civil, sobre las que se analizan distintas características, comportamientos y propiedades de determinados elementos, entre las que más se destacan están: i) Análisis de estructuras, se encarga de estimar la resistencia máxima de elementos 
sometidos a cargas vivas, cargas muertas y cargas accidentales (sismos, vientos, nieve, etc.); ii) Análisis geotécnico, se encarga de estimar la resistencia entre partículas de la corteza terrestre de distinta naturaleza, granulometría, humedad, cohesión, y de las propiedades de los suelos en general, con el fin de asegurar la interacción del suelo con la estructura. iii) Análisis hidráulico, Se ocupa de la proyección y ejecución de obras relacionadas con el agua, sea para su uso, como en la obtención de energía hidráulica, la irrigación, canalización u otras, sea para la construcción de estructuras en mares, ríos, lagos, o entornos similares, incluyendo, por ejemplo, diques, represas, canales, puertos, muelles, rompeolas, entre otras construcciones. También hace referencia a las máquinas hidráulicas; iv) Análisis de materiales, se encarga del análisis, estudia las propiedades de los materiales utilizados en la construcción de obras civiles como el concreto hidráulico, el concreto asfáltico, el acero y los polímero, entre muchos otros.

Cada una de las disciplinas, implementan laboratorios y actividades experimentales que especifican la observación, el proceso y los resultados, de los análisis de la actividad científica que puede darse sobre los elementos asociados en cada disciplina. En los laboratorios, las prácticas experimentales aportan, parte del método científico, validan la teoría y generan nuevos hallazgos. Las principales prácticas de laboratorio, del dominio de la Ingeniería Civil, están agrupadas en las siguientes categorías.

Laboratorio de materiales: Se denomina ensayo de materiales a toda prueba cuyo fin es determinar las propiedades mecánicas de un material. En este laboratorio, se estudia el comportamiento y propiedades de materiales de construcción comúnmente usados en aplicaciones de Ingeniería Civil tales como concreto, acero, madera, piezas de mampostería, y plásticos. Normas y estándares que describen estos materiales y los ensayos para determinar sus propiedades. Prácticas de laboratorio para el ensayo y estudio de diferentes materiales. Ciencia de los materiales, teorías de falla, flujo plástico, relajación, fatiga, concentración de esfuerzos, vibraciones.

Laboratorio de mecánica de fluidos: El objetivo del laboratorio es tratar el tema de los fluidos desde el punto de vista de sus propiedades físicas y su comportamiento mecánico. Al final del curso el estudiante estará en capacidad de entender el comportamiento de los fluidos en diferentes situaciones de Ingeniería, con base en la aplicación de las ecuaciones de la física y de métodos numéricos para facilitar los cálculos. Cita experimentos de énfasis en las pérdidas por fricción y su efecto sobre el diseño de sistemas de Ingeniería relacionados con el manejo del recurso agua. Cubre también análisis experimentales de: la estática de los fluidos, la cinemática de los fluidos, las propiedades de los fluido y el comportamiento de los fluidos reales (esfuerzos cortantes, distribución de velocidades, pérdidas de energía), análisis dimensional y similitud dinámica, flujo en sistemas de tuberías a presión, diseño de tuberías, aplicaciones.

Laboratorio de Suelos: Se ocupa de valorar la composición y características de los suelos tienen como fin llegar a explicaciones físicas y descripciones matemáticas del comportamiento del suelo cuando es sometido a cargas monotónicas, haciendo especial énfasis en la respuesta no lineal del suelo

\section{ONTOLOGIAS PARA MODELAR EXPERIMENTOS}

Describir formalmente experimentos para el análisis, anotación y eficiencia en el intercambio de los resultados científicos, ha sido una tarea, previamente realizada por varios científicos. Entre ellas se destacan: EXPO, P-PLAN y EXACT.

EXPO [18], es una ontología la cual se basa en las ideas de la filosofía de la ciencia (lógica, probabilística, metodológico, epistemológico, etc.) Esta describe el conocimiento general sobre el mundo, analiza ontologías existentes como las bioontologías y teorías como el diseño de experimentos; esta ontología propone conocimiento en niveles apropiados de la abstracción la que lo hace que sea factible y deseable ya que en los principios experimentales se usa para hacer un análisis eficiente, anotación y el intercambio de resultados lo cual es un objetivo en la ciencia. EXPO es un desarrollo que intenta crear una ontología general para la experimentación científica.

P-PLAN [12], una extensión de la ontología PROV-O el cual expresa el modelo de datos que "Proporciona un conjunto de clases, propiedades y restricciones que se pueden utilizar para representar e intercambiar información de procedencia generada en diferentes sistemas y en diferentes contextos" [24]. P-PLAN se desarrolló como una ontología que describe el flujo de trabajo científico abstracto como los planes la cual da un registro de afirmaciones acerca de los pasos y procedencia que describe al ejecutarse. ésta diseñada para describir ensayos ya que estos son específicos a la hora de hacer una investigación, también es importante la organización de los objetos de investigación, publicar como se planearon y realizaron los experimentos científicos los cuales PROV no los describía explícitamente; gracias a esto P-plan da la oportunidad junto con PROV de crear una ontología que resolviera este problema y diera la oportunidad de mostrar planes en ejecución superior y también plan en la parte inferior.

EXACT [19], es una ontología que actúa, como base metódica para representar el protocolo experimental de un laboratorio biológico y en bioinformática. EXACT proporciona un modelo para la descripción de las acciones del experimento, y puede ser utilizado para la representación totalmente formalizada de protocolos experimentales, o se puede 
combinar con otros formalismos para la descripción de las investigaciones biomédicas y así hacerlas reproducibles.

La Tabla I, constituye un resumen comparativo de las ontologías experimentales citadas. Sin embargo, estas no resultan las únicas, ya que la literatura, aporta otras que apuntan al mismo objetivo [2, 14], sin embargo las citadas han sido elegidas por su generalidad.

\section{TABLA I. ONTOLOGÍAS PARA REPRESENTAR EL DOMINIO EXPERIMENTAL}

\section{CUADRO RESUMEN DE ONTOLOGIAS PARA DESCRIBIR UN EXPERIMENTO CIENTÍFICO}

\begin{tabular}{|c|c|c|c|}
\hline DEFINICION & \multicolumn{3}{|c|}{$\begin{array}{l}\text { Una ontología es leíble tanto por humanos } \\
\text { como por máquinas. Y representa un conjunto } \\
\text { de conceptos con una sintaxis y semántica, } \\
\text { provista en un lenguaje de representación de } \\
\text { conocimiento. }\end{array}$} \\
\hline $\begin{array}{c}\text { CLASES DE } \\
\text { ONTOLOGIA } \\
\text { S }\end{array}$ & EXPO & P-PLAN & EXACT \\
\hline $\begin{array}{l}\text { TEMA DE LA } \\
\text { ONTOLOGIA }\end{array}$ & $\begin{array}{c}\text { Esta describe } \\
\text { el } \\
\text { conocimiento } \\
\text { general sobre } \\
\text { el mundo, } \\
\text { analiza } \\
\text { ontologías } \\
\text { existentes } \\
\text { como las bio- } \\
\text { ontologías y } \\
\text { teorías para el } \\
\text { diseño de } \\
\text { experimentos. } \\
\text { Es una } \\
\text { ontología que } \\
\text { pretende ser } \\
\text { general para la } \\
\text { descripción de } \\
\text { experimentaci } \\
\text { ón científica. }\end{array}$ & $\begin{array}{c}\text { Es una } \\
\text { extensión de } \\
\text { la ontología } \\
\text { PROV-O. P- } \\
\text { PLAN } \\
\text { describe flujo } \\
\text { de trabajo } \\
\text { científico } \\
\text { abstracto } \\
\text { como los } \\
\text { planes la cual } \\
\text { da un registro } \\
\text { de } \\
\text { afirmaciones } \\
\text { acerca de los } \\
\text { pasos y } \\
\text { procedencia } \\
\text { que describe } \\
\text { al ejecutarse. }\end{array}$ & $\begin{array}{c}\text { La ontología } \\
\text { EXACT es } \\
\text { la base de } \\
\text { un método } \\
\text { de } \\
\text { representar } \\
\text { protocolo de } \\
\text { laboratorio } \\
\text { biológico y } \\
\text { en la } \\
\text { bioinformáti } \\
\text { ca. }\end{array}$ \\
\hline $\begin{array}{l}\text { PORQUE SE } \\
\text { CREARON }\end{array}$ & $\begin{array}{l}\text { Se realizó con } \\
\text { el objetivo de } \\
\text { describir } \\
\text { formalmente } \\
\text { experimentos } \\
\text { para el } \\
\text { análisis, } \\
\text { anotación y } \\
\text { eficiencia en } \\
\text { el intercambio } \\
\text { de los } \\
\text { resultados } \\
\text { científicos. }\end{array}$ & $\begin{array}{c}\text { Se creó como } \\
\text { una solución a } \\
\text { la } \\
\text { organización } \\
\text { de la } \\
\text { información, } \\
\text { la publicación, } \\
\text { planeación y } \\
\text { realización de } \\
\text { los } \\
\text { experimentos } \\
\text { científicos en } \\
\text { manera de } \\
\text { ensayos lo }\end{array}$ & $\begin{array}{c}\text { Se creó } \\
\text { como la } \\
\text { base de un } \\
\text { método de } \\
\text { para } \\
\text { representar } \\
\text { protocolos } \\
\text { de } \\
\text { laboratorio } \\
\text { biológicos, } \\
\text { dando así } \\
\text { una solución } \\
\text { a los } \\
\text { investigador }\end{array}$ \\
\hline
\end{tabular}

\begin{tabular}{|c|c|c|c|}
\hline & & $\begin{array}{c}\text { cual hace una } \\
\text { definición } \\
\text { más explícita. }\end{array}$ & $\begin{array}{c}\text { es los } \\
\text { medios para } \\
\text { describir los } \\
\text { ensayos } \\
\text { acerca de } \\
\text { este tema. }\end{array}$ \\
\hline DOMINIO & bio-ontologías & Biogenética & $\begin{array}{c}\text { biológico y } \\
\text { en la } \\
\text { bioinformáti } \\
\text { ca }\end{array}$ \\
\hline LENGUAJE & W3C OWL & W3C OWL2 & W3C OWL \\
\hline
\end{tabular}

\section{REPRESENTACION SEMÁNTICA DE UN PROTOCOLO EXPERIMENTAL EN CIVIL}

Un protocolo experimental en cualquier disciplina de la ingeniería Civil corresponde a una práctica de laboratorio, direccionada. La Figura 1, muestra una representación real de un protocolo experimental, en este caso el de suelos, de la Universidad Nacional de México [21].

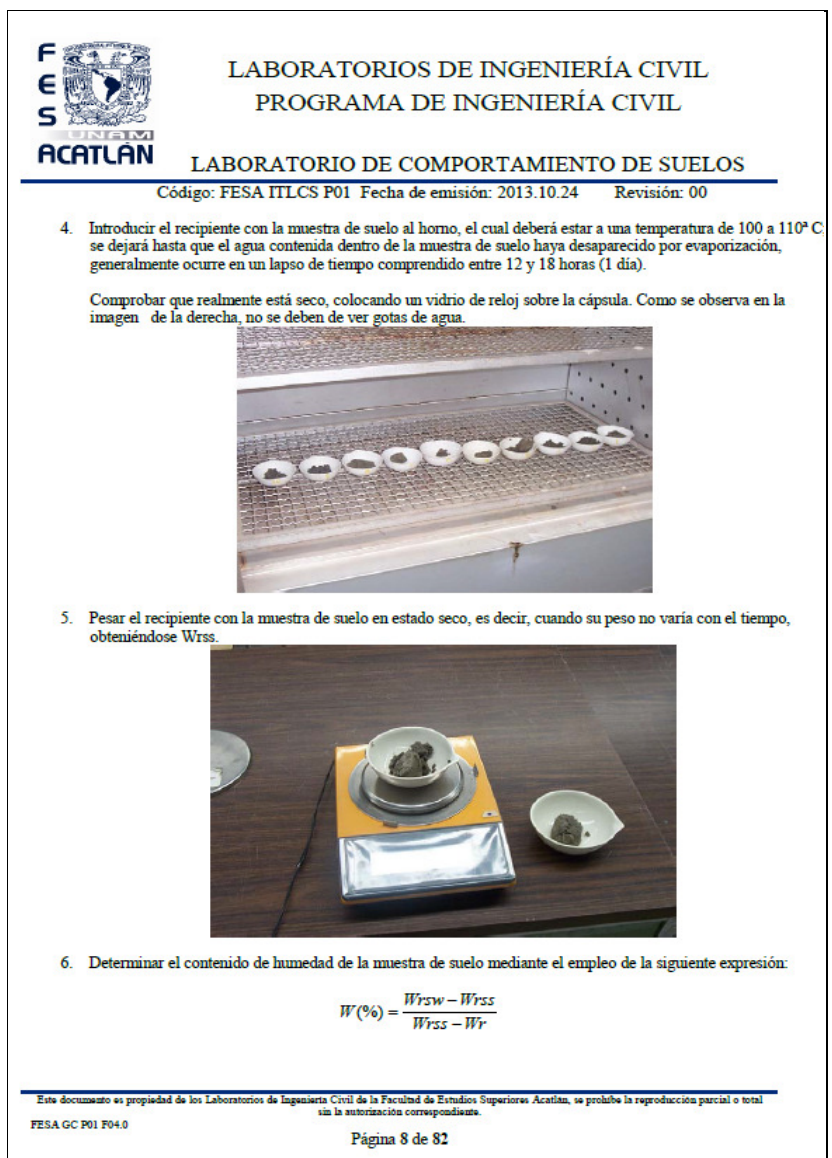

Figura 1. Ejemplo de un protocolo experimental, Laboratorio de Suelos. Fuente: Universidad Aclatan, Programa Ingenieria Civil [21]

Sin embargo, y cómo es posible apreciar en la Figura 2, el protocolo experimental muestra la representación abstracta de 
cada uno de los elementos que pueden ser incluidos en un protocolo experimental. Aunque la Figura 3, reconoce dos macro elementos, claves: una estructura documental que representa los pasos del método científico y una descripción del paso a paso, descrito mediante un flujo de procesos (conocido como workflow-científico) [1, 11, 5]. La descripción anterior, es fiel reflejo de la presentada en EXACT, la cual está diseñada para el dominio de la biotecnología de las plantas vegetales. El objetivo de modelar el protocolo en dos partes, separando el modelo de información estático, del modelo dinámico. Siendo el primero representado como un documento científico, que registra la información básica de la metodología de la investigación; mientras, el segundo, representa el protocolo como un flujo de procesos [7]. En esta representación, es posible modelar la guía del laboratorio, como la especificación abstracta del proceso experimental (workflow científico), que debe seguirse para alcanzar los objetivos, propósitos y/o resultados reportados por ese experimento (documento científico).

Cuando se habla de reutilizar el conocimiento, independiente del dominio, resulta clave [25]: i) la comprensión común de la estructura de información entre personas y agentes de software, ii) la representación de manera explícita de los supuestos de un dominio, y finalmente, iii) la disociación del conocimiento de un dominio, del conocimiento que se pueda denominar operacional. En otras palabras, se habla de generar una estructura semántica, como ontología que soporte los aspectos claves de la reutilización; que para este caso se concentra en la especificación del conocimiento de los protocolos experimentales del dominio de la Ingeniería Civil.

La descripción formal de los experimentos es necesaria para lograr un análisis eficiente de la investigación y permitir el intercambio de resultados científicos, constituyéndose en una parte fundamental de la práctica de la ciencia [20]. Se requieren ontologías para lograr este objetivo. Representar semánticamente el dominio de los protocolos experimentales del campo de la ingeniería civil, permite contar con un esquema único para modelar la investigación científica del área.

Tal representación debe ser univoca, consensuada y debe contener los elementos comunes, necesarios y suficientes, para lograr reutilizar los experimentos, sin importar quién o para que los requiera. Con el objetivo de proponer una ontología para este campo, se formulan varias propuestas como guías metodológicas, pero, se recurre a una de las más comúnmente aceptada Ontology Development 101” [15], en donde sus principales recomendaciones consisten en: i) determinar el dominio y ámbito de la ontología, ii) determinar la intención de uso de la ontología, iii) reutilizar ontologías o vocabularios controlados existentes; iv) enumerar los términos importantes del dominio; v) definir jerarquía de clases; vi) crear las instancias determina las actividades que deben considerarse para generar una nueva ontología.
Aunque aparente la cuestión de determinar el dominio estaba clara, el tema de los protocolos experimentales en Ingeniería Civil, presenta una amplia categorización de elementos y conceptos, razón por la cual establecer un límite de profundización para cada rama fue una tarea muy cuidadosa aunque poco justa, ya que cada rama podía generar otra ontología completa.

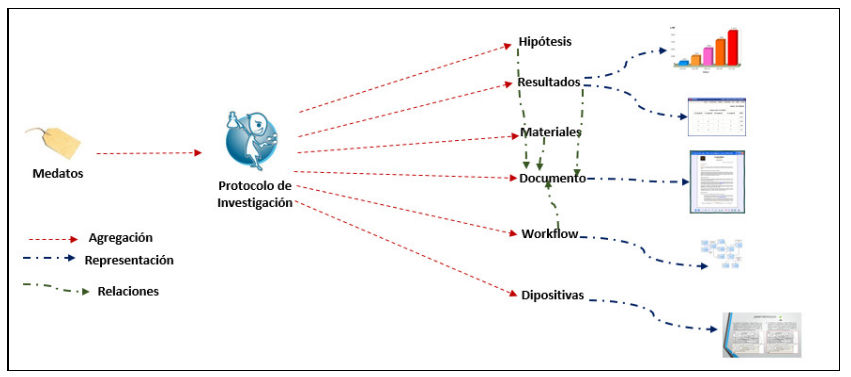

Figura 2. Ejemplo abstracto de un protocolo experimental

La definición de cada categoría temática, fue el reflejo de la labor de consenso llevada a cabo entre el análisis de varios protocolos experimentales (basados en la descripción que se definió en la Figura 3) y los expertos en el tema (Ingenieros y científicos). Como resultados las áreas temáticas en nuestra ontología se limitan a la definición de categorías genéricas por tema y subtemas. La intención de uso de la ontología, era de las tareas más claras hasta ahora: la clasificación y reutilización del conocimiento que se genere en los laboratorios del área de Ingeniería Civil. Por ello, la recomendación en la que más se centró la atención, fue precisamente en la reutilización de ontologías existentes, así que a la hora de considerar la reutilización de estándares de tipo ontológico en este campo, se siguieron propuestas bajo un esquema de modelar experimentos, similares a P-PLAN, EXPO y EXACT. Adoptar ontologías de existencia previa, contribuye a definir los conceptos más importantes y comunes del dominio experimental; sin embargo, algunos de tales conceptos, resultan repetidos, mientras que otros pueden ser insuficientes o incluso innecesarios para modelar el conocimiento de la ingeniería Civil. Dadas las circunstancias anteriores, la primera, actividad de esta etapa, consistió en determinar los elementos (datos e información) registrados en los contenidos de los protocolos. Esta tarea estuvo inspirada en la formulación de la Figura 3, la cual se estableció el detalle de cada protocolo, del área de la ingeniería civil y su representación bajo la composición de las dos partes citadas: documento y workflow.

Para enumerar los términos más importantes del dominio, se decidió representar cada elemento abstracto de los principales protocolos de ingeniería civil, como instancias de la representación, mostrada en la Fig. 2. Generándose con ello, la Fig. 3, la cual proporciona elementos instanciados, para cada clase de las ontologías experimentales reutilizadas. El proceso, señalado en esa Fig. 3, es repetido para cada uno de los protocolos experimentales reportados en el dominio de la 
ingeniería civil. La ontología construida, se encuentra conformada por los principales conceptos jerarquizados, asociados a los protocolos de ingeniería civil. Tal como se muestra en la Fig. 4. Sin embargo, se ha decido importar la ontología EXPO, descrita en descrita en Tabla I.

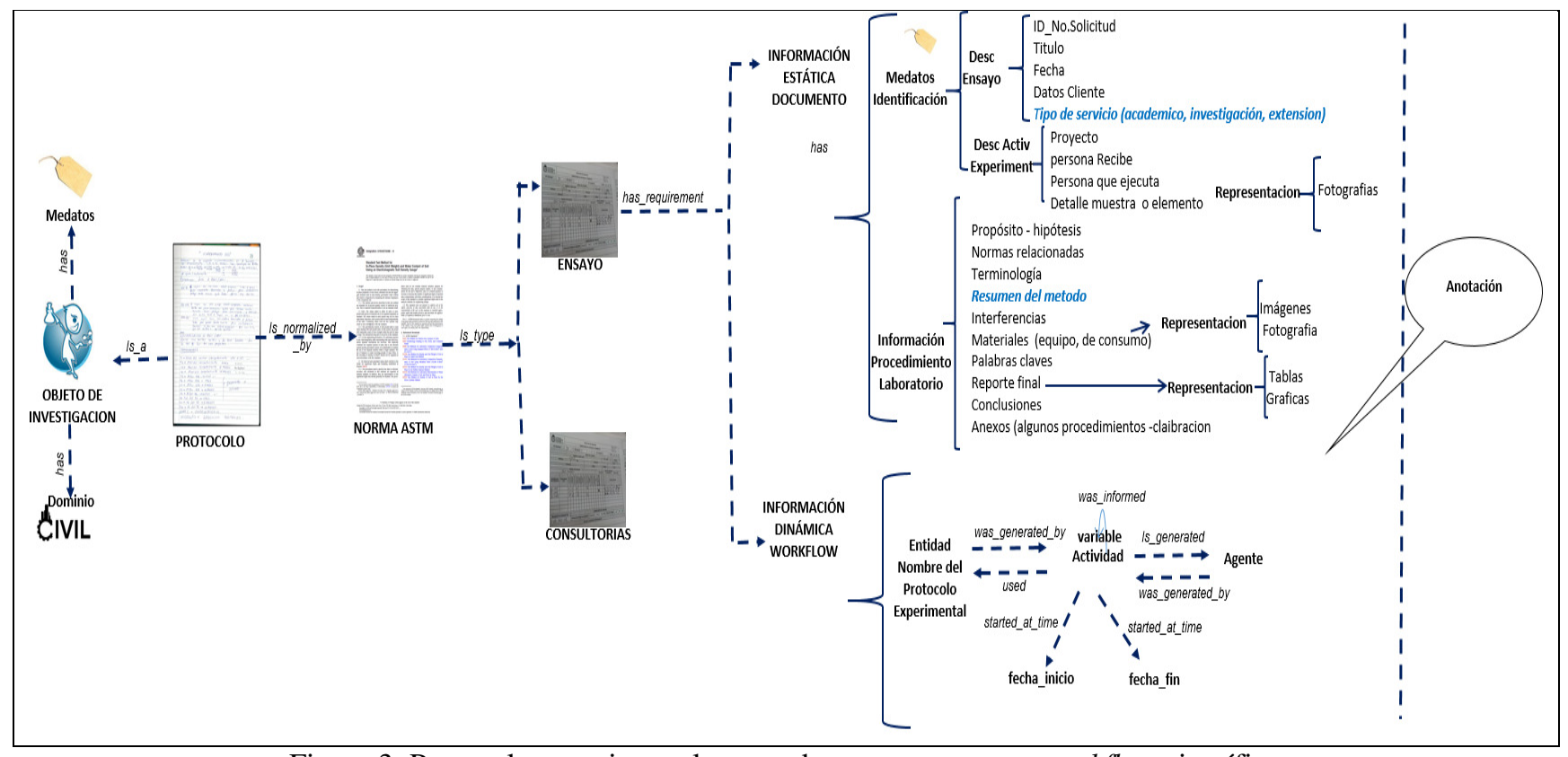

Figura 3. Protocolo experimental, como documento y como workflow científico 



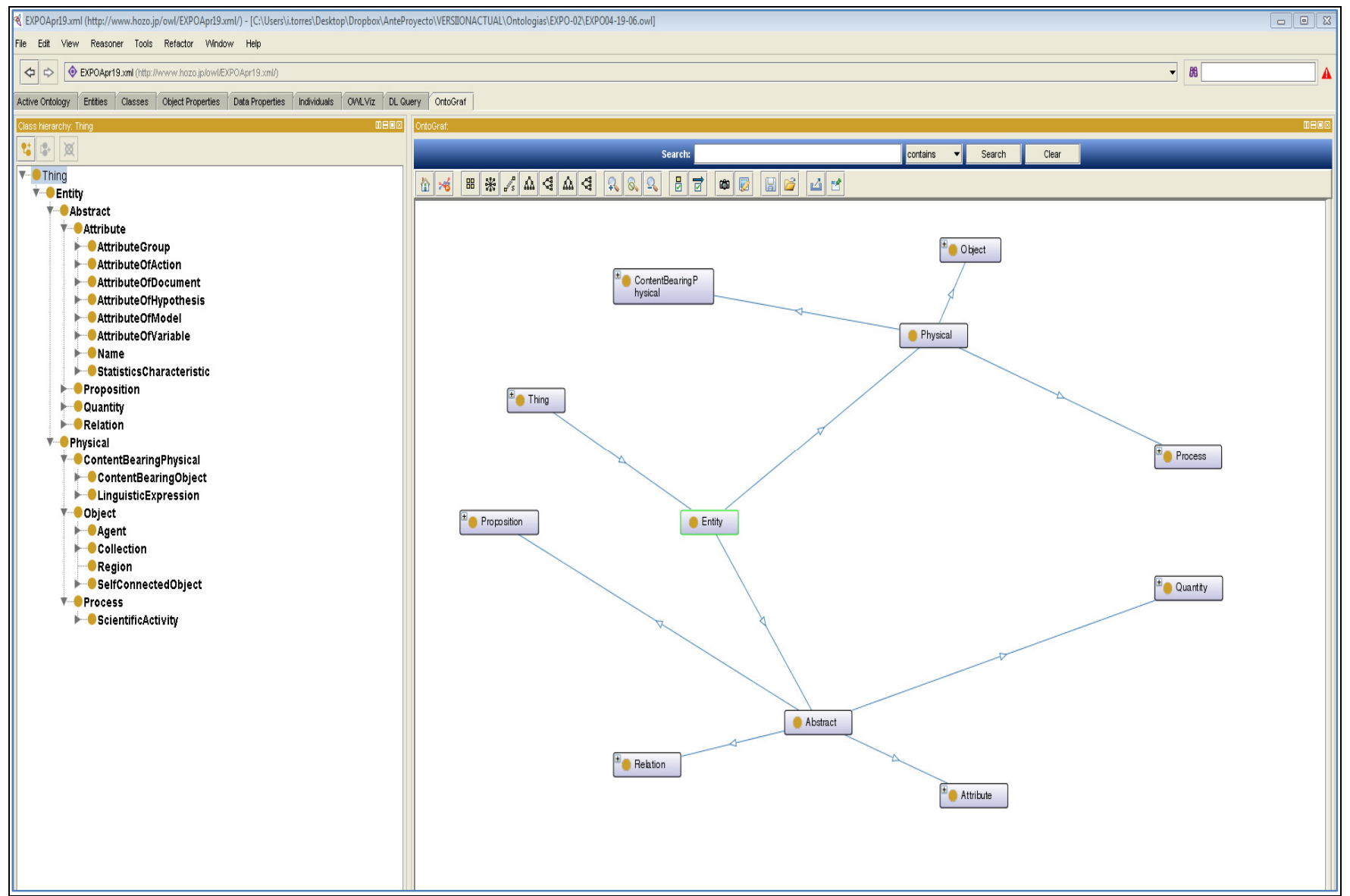

Figura 4. Protocolo experimental vista semántica

experimentales de las áreas de la ingeniería civil, citadas en la sección II, numeral C. Sin embargo, se espera enriquecer el modelo con la incorporación de un mecanismo que permita

\section{CONCLUSIONES}

Actualmente los protocolos de laboratorio, en el campo de la ingeniería civil (al igual que en muchos otros dominios) recopilan toda la información científica de un experimento. Tal información, resulta clave cuando se decide extender el límite del conocimiento en esa área. Sin embargo, no contar con un mecanismo que formalice su representación univoca, carente de ambigüedad, es uno de los graves impedimentos que existe en el ambiente del conocimiento científico.

Conscientes la anterior limitante, éste articulo presenta una propuesta para formalizar el conocimiento científico, registrado en los protocolos experimentales del área de ingeniería civil, convirtiéndose en el aporte principal de este trabajo. A la fecha, el área de la ingeniería civil no ha sido aún formalizada para ser reutilizada y reproducida, de la manera en que lo establece el paradigma de e-sicence, careciendo de una interpretación comprensible y autónoma de los protocolos experimentales tanto por humanos como por parte de las máquinas.

Como trabajo futuro, el modelo de representación actual, está siendo extendido, a incorporar los principales protocolos

\section{RECOMENDACIONES}

Este trabajo presenta resultados preliminares del proyecto "Un Modelo Semántico para la Representación de Protocolos Experimentales en el Área de Ingeniería Civil" -Convocatoria Programa Nacional de Apoyo A Estudiantes de Posgrado para el Fortalecimiento de la Investigación, Creación e Innovación adscrito a la Universidad Nacional de Colombia-, identificado con código Quipu 200000013687.

\section{REFERENCIAS}

[1]. P. Alper, K. Belhajjame y C. Goble. (2013). Small is beautiful: summarizing scientific workflows using semantic annotations. Big Data.

[2]. R. Arp y B. Smith. (2008). BFO: Function, role, and disposition in basic formal ontology. Disponible: Nature Precedings. http://precedings.nature.com/documents/1941/versio n/1/files/npre20081200-1.pdf. 
[3]. ASTM. International - Standards Worldwide. Disponible en http://www.astm.org/

[4]. S. Bechhofer, D. Roure, y M. Gamble. (2010). Research objects: Towards exchange and reuse of digital knowledge: precedings.nature.com.

[5]. S. Bechhofer, S. Soiland-Reyes, y K. Belhajjame. (2011). Workflow Lifecycle Management Initial Requirements., SWAT4LS, London, 2011.

[6]. K.. Belhajjame. (2007). Semantic replaceability of eScience web services. E-Science and Grid Computing. IEEE International Conference on eScience and Grid Computing, Pages 449-456, IEEE CS, 2007.

[7]. K. Belhajjame, O. Corcho, D. Garijo, y J. Zhao. (2012). Workflow-centric research objects: First class citizens in scholarly discourse: users.ox.ac.uk. in n proceedings of the ESWC2012 Workshop on the Future of Scholarly Communication in the Semantic Web (SePublica2012), Heraklion, Greece, May 2012

[8]. K. Belhajjame, C. Globe. (2012). Research object management: opportunities and challenges. Paper presented at the Conference on Computer Supported Cooperative Work. CSCW 2012, Washington, USA.

[9]. J. H. Berners-Lee, y O. Lassila, (2001). The semantic web. Scientific American, 5, 284. O. Corcho (2015). E-ciencia semántica. http://mayor2.dia.fi.upm.es/oegupm/index.php/es/researchareas/3-semanticscience.

[10]. M. Courtot, W. Bug, F. Gibson, Al. L. Lister, J. Malone, D. Schober, R. R. Brinkman y A. Ruttenberg. (2008). OBI: The OWL of Biomedical Investigations. http://sourceforge.net/p/obi/code/HEAD/tree/.

[11]. E. Deelman, D. Gannon, M. Shields, y I. Taylor. (2009). Workflows and e-science: An overview of workflow system features and capabilities. Future Generation Computer Systems, 25(5), 12

[12]. D. Garrijo y Y. Gil. (2012). P-PLAN Ontology. http://www.opmw.org/model/p-plan10112012/

[13]. O. Giraldo. (2011). Manejo del Conocimeinto en los cuadernos de laboratorio. Tesis de Maestria, facultad de Ciencias Agrarias, Universidad Nacional de Colombia, sede Palmira.

[14]. O. Giraldo. (2014). SMART Protocols Ontology. http://vocab.linkeddata.es/SMARTProtocols/spworkflow.htm

[15]. F. Natalya L. McGuinness. (2006). Ontology Development 101: A Guide to Creating Your First Ontology. Disponible http://protege.stanford.edu/publications/ontology_de velopment/ontology101.pdf

[16]. RAE. (2015). Real Academia Española. Diccionario. Madrid España. Disponible http://www.rae.es/

[17]. D. Roure, C. Goble, S. Aleksejevs, y S. Bechhofer. (2009). The myexperiment open repository for scientific workflows: eprints.soton.ac.uk.
[18]. L. Soldatova y R. King. (2006). An ontology of scientific experiments. Journal List. J. R. Soc Interface. V3(11). doi: 10.1098/rsif.2006.0134. Disponible http://www.ncbi.nlm.nih.gov/pmc/articles/PMC1885 $356 /$.

[19]. L. Soldatova, W. Aubrey y R. King. (2006). The EXACT description of biomedical protocols. Oxford JournalsScience \& Mathematics Bioinformatics Volume 24, Issue 13Pp. 295-303.

[20]. Y. Sun, J. Lizhe, F. Wei, F. Cheng. (2011). Research in e-Science: Current Status and Future Directions. [Editorial]. International Journal of Computer Science and Engineering, 2011.

[21]. Universidad Nacional Autónmoma de México, Laboratorios de Ingeniería Civil. Programa de Ingneiría Civil. Mnaul de prácticas de Laboratorio. http://www.acatlan.unam.mx/licenciaturas/30/

[22]. Universidad Nacional de Colombia. (2000). Laboratorios Universidad Nacional de Colombia, sede Medellín. Facultad de Minas. Retrieved Abril, 2014, from http://www.minas.medellin.unal.edu.co/dirlab/index. php/laboratorios

[23]. L. Verdes-Montenegro. (2012). e-Science for the SKA, WF4Ever: Supporting Reuse and Reproducibility in Experimental Science RadioNet Advanced Radio Astronomy, Commissioning Skills and Preparation for the SKA Manchester: November 15th 2012

[24]. X. Yang, J. Lizhei, J. Wei. (2011). Guide to eScience Next Generation Scientific Research and Discovery. Springer 2011. doi: 0.1007/978-0-85729439-5

[25]. J. Zhao, G. Klyne, P. Holubowicz, y R. Palma. (2012). RO-Manager: A Tool for Creating and Manipulating Research Objects to Support Reproducibility and Reuse in Sciences: ceur-ws.org. 